\title{
Grow your own valve
}

\author{
$\left\{\begin{array}{l}010 \\ \left(\frac{1 x}{2}\right)\end{array}\right.$ \\ Michael Rahbek Schmidt, MD, PhD, DMSc; Lars Søndergaard*, MD, DMSc \\ Rigshospitalet University Hospital, Copenhagen, Denmark
}

Implantation of bioprosthetic valves or conduits notoriously implies concerns about limited durability and, in children, the risk of outgrowing the prosthesis causing the likely need for reintervention. The degenerative processes leading to failing function of implanted grafts are unpredictable and the longevity varies extensively. Aside from progressive symptom burden, graft deterioration also entails increased risk of infection, arrhythmia and even sudden death. The aspiration of more durable bioprosthetic materials has driven research for decades, but development of novel materials with reduced triggering of chronic inflammation and improved resilience towards calcification has so far been limited.

Transcatheter aortic valve implantation (TAVI) utilising stent valves with glutaraldehyde-fixed animal-derived tissue has increasingly become a favoured treatment option in patients with aortic valve stenosis and increased surgical risk. This is supported by recent trials and meta-analyses, which show similar or superior outcomes compared to surgical aortic valve replacement ${ }^{1-3}$. However, as the use of TAVI has soared globally, so have anxieties about long-term valve durability, in particular as TAVI is progressively being adopted in younger lower-risk patient groups with longer life expectancy. The use of bioprosthetic aortic valves in younger patients has also been embraced by cardiac surgeons, partly driven by patient preference to avoid anticoagulation therapy. While valve-in-valve TAVI is emerging as a potential treatment for failing bioprosthetic valves, the long-term outcome of this novel therapy is uncertain.

The paediatric patient population undergoing implantation of bioprosthetic valves and valved conduits encompasses, e.g., congenital aortic valve stenosis (Ross procedures), tetralogy of Fallot and transposition of the great arteries. These patients often receive a homograft or other right ventricle-to-pulmonary artery (RV-PA) valved conduit at a young age and thus pose the specific challenge of growth besides a long post-procedural lifespan, which itself often leads to the need for repeated change of the implanted bioprosthesis. A non-degenerating biocompatible conduit with the ability to grow is therefore of particular interest in paediatric patients.

In this issue of EuroIntervention, two papers describe how a novel technology based on cell-free absorbable scaffolds can potentially be used to overcome some of the limitations of traditional bioprosthetic grafts. Both studies are based on the hypothesis

*Corresponding author: Kardiologisk Klinik B 2011, Rigshospitalet University Hospital, Blegdamsvej 9, 2100 Copenhagen,

Denmark.E-mail: Lars.Soendergaard.01@regionh.dk 
that functional restoration is obtainable through recruitment of the recipient's own cells to replace the biodegradable porous polymeric scaffold gradually, so-called Endogenous Tissue Restoration (ETR).

Miyazaki et al have investigated the acute performance of the XELTIS aortic valve (Xeltis BV, Eindhoven, the Netherlands), a first-generation stent valve with absorbable polymeric leaflets, in adult sheep 4 .

\section{Article, see page 1410}

Using echocardiography and videodensitometric analysis, the authors show that the acute haemodynamic performance of the valve is similar to standard commercially available TAVI valves. While the focus of the paper is on the implantation technique and immediate mechanical performance of the valve, the most important finding in the study may be that the porous polymeric valve leaflets apparently serve well as fully functioning leaflet "tissue", at least in the acute setting. However, long-term results are awaited.

In the second paper, Soliman et al compare the six-month performance of the XELTIS biodegradable valved conduit (Xeltis $\mathrm{BV})$ to a standard commercially available valved conduit made from a porcine aortic valve in a woven fabric tube (Hancock ${ }^{\circledR}$ conduit; Medtronic, Minneapolis, MN, USA) and furthermore investigate the 24-month performance of the XELTIS pulmonary valve (XPV) conduit ${ }^{5}$.

\section{Article, see page 1418}

The conduits were implanted in supravalvar interposition pulmonary artery vascular grafts (i.e., not as true RV-PA conduits) in adult sheep. The haemodynamic performance of the two conduits was similar at six months (low pressure gradient and only trace or mild central regurgitation) measured by echocardiography, but curiously the XPV conduit diameter increased gradually over the 24 -month follow-up period up to $150 \%$ of its original diameter, which is described as a "positive conduit patency index" and apparently occurred without growth of the sheep, which were already adult at the time of implantation. Growth of an implanted RV-PA conduit may have obvious potential clinical benefits in smaller children if the increase in size is slow and similar to the natural growth of the normal main pulmonary artery; however, uncontrolled growth/dilatation may cause problems that outweigh the benefits, such as risk of rupture and compression of surrounding structures. Further bioengineering work may be required to develop a vascular graft of biodegradable material that only allows limited growth. Bockeria et al have tested an unvalved conduit of similar material as a cavopulmonary connection in Fontan operations in humans with a follow-up period of 12 months with no reported signs of expansion of the conduit ${ }^{6}$, which may indicate that the growth of the XPV conduit is induced by the pulsation or pressure in pulmonary circulation compared to low near-constant pressure in the Fontan circulation.
In line with other recent discoveries, such as the potential clinical use of intestinal microbiome and invention of new biomimetic pharmacological compounds, ETR takes advantage of the mammalian body's ability to restore and repair itself, and this concept could hold great promise. Future clinicians may consider the current approach of implanting metallic non-degradable foreign bodies in patients somewhat primitive, and the absence of foreign material in the circulation may also diminish the need for antithrombotic therapy. However, as the currently used bioprosthetic valves and conduits are also undergoing development and refinement, and TAVI valves in particular are improving rapidly, competition is strong, and extensive testing in clinical trials is mandated if these exciting novel technological advances are to find their way into clinical use.

\section{Conflict of interest statement}

The authors have no conflicts of interest to declare.

\section{References}

1. Thyregod HG, Steinbruchel DA, Ihlemann N, Nissen H, Kjeldsen BJ, Petursson P, Chang Y, Franzen OW, Engstrøm T, Clemmensen P, Hansen PB, Andersen LW, Olsen PS, Søndergaard L. Transcatheter Versus Surgical Aortic Valve Replacement in Patients With Severe Aortic Valve Stenosis: 1-Year Results From the AllComers NOTION Randomized Clinical Trial. J Am Coll Cardiol. 2015;65:2184-94.

2. Franzone A, Piccolo R, Siontis GC, Lanz J, Stortecky S, Praz F, Roost E, Vollenbroich R, Windecker S, Pilgrim T. Transcatheter Aortic Valve Replacement for the Treatment of Pure Native Aortic Valve Regurgitation: A Systematic Review. JACC Cardiovasc Interv. 2016;9:2308-17.

3. Siontis GC, Praz F, Pilgrim T, Mavridis D, Verma S, Salanti G, Søndergaard L, Jüni P, Windecker S. Transcatheter aortic valve implantation vs. surgical aortic valve replacement for treatment of severe aortic stenosis: a meta-analysis of randomized trials. Eur Heart J. 2016;37:3503-12.

4. Miyazaki Y, Soliman OI, Abdelghani M, Katsikis A, Naz C, Lopes S, Warnack B, Cox M, Onuma Y, Serruys PW. Acute performance of a novel restorative transcatheter aortic valve: preclinical results. EuroIntervention. 2017;13:e1410-7.

5. Soliman OI, Miyazaki Y, Abdelghani M, Brugmans M, Witsenburg M, Onuma Y, Cox M, Serruys PW. Midterm performance of a novel restorative pulmonary valved conduit: preclinical results. EuroIntervention. 2017;13:e1418-27.

6. Bockeria LA, Svanidze O, Kim A, Shatalov K, Makarenko V, Cox M, Carrel T. Total cavopulmonary connection with a new bioabsorbable vascular graft: First clinical experience. J Thorac Cardiovasc Surg. 2017;153:1542-50. 